\title{
Sur Lutzomyia davisi (Root, 1934) et les espèces de morphologie comparable (Diptera, Phlebotomidae)
}

\author{
par E. ABONNENC *, J. ARIAS **, N. LEGER ${ }^{* * *}$ et D.G. YOUNG **** \\ * 4, passage Catinat, F 95210 Saint-Gratien. \\ * Instituto Nacional des Pesquisas de Amazonia, Manaus, Brésil. \\ *** U.E.R. de Pharmacie de Reims, F 51096 Reims. \\ **** Université de Floride (Entomology), 3103 McCarty Hall, Gainesville 3261l, U.S.A.
}

\begin{abstract}
RESUME. Après examen de nombreux exemplaires sud-américains de «Lutzomyia davisi », les auteurs ont été amenés à distinguer, à côté de l'espèce Lutzomyia davisi (Root, 1934), deux espèoes voisines : L. robini n. sp. et L. claustrei Abonnenc, Léger et Fauran, 1979. Ils décrivent ici le mâle et la femelle de la première espèce et la femelle de la seconde. Ils donnent en outre une redescription de $L$. davisi ainsi que la distribution, telle qu'elle est connue à ce jour, des trois espèces en Guyane française et au Brésil.
\end{abstract}

On Lutzomyia davisi (Root, 1934) and some related species (Diptera, Phlebotomidae).

SUMMARY. Lutzomyia davisi (Root, 1934) and two related South American species are distinguished. Both sexes of $\mathrm{L}$. robini $n$. sp. and the female of $\mathrm{L}$. claustrei Abonnenc, Léger et Fauran, 1979 are described and illustrated for the first time. A redescription of both sexes of $\mathrm{L}$. davisi is provided. The known distribution in French Guiana and Brazil for each species is given.

Une étude minutieuse des Phlébotomes appartenant au sous-genre Psychodopygus, de type «Lutzomyia davisi » récoltés en Guyane française, conduisait deux d'entre nous à distinguer au sein de ce groupe deux espèces voisines: $L$. davisi (Root, 1934) et $L$. claustrei n. sp. (Abonnenc et coll., 1979).

Depuis, ces deux espèces ont été retrouvées au Brésil (collections Young et Arias) et leur validité confirmés.

Accepté le 5 mai 1980. 
L'examen de tous ces exemplaires, et leur étude comparée, devait nous permettre, en outre, d'individualiser, au sein du même groupe, une troisième espèce.

Nous donnons ici :

- une redescription du mâle de L . davisi,

- une description du mâle de la nouvelle espèce que nous avons nommée : Lutzomyia robini en hommage au $\mathrm{D}^{r}$ Robin, directeur de l'Institut Pasteur de Cayenne dans le cadre duquel une partie de ce travail a été réalisée,

- un rappel des caractères morphologiques spécifiques de $L$. claustrei et une justification de son individualisation,

- pour chacune de ces trois espèces, une description de la femelle présumée, en fonction des associations observées dans les divens lots cie phlébotomes examinés.

\section{Lutzomyia davisi (Root, 1934)}

$=$ L. rooti $($ Mangabeira, 1942) [Young, 1979]

La description de ce Phlébotome, donnée par Root, est assez sommaire, mais l'auteur donne une très bonne figure de l'armature génitale dont chaque paramère porte, sur son bord inférieur, 17 soies foliacées régulièrement réparties et diminuant progressivement de longueur, de la base vers l'apex. Il indiqus, en outre, les « wings ratio » a., $\beta, \gamma$ et $\delta$ de l'aile et la longueur des segments des palpes.

Le caractère qui, comparé à nos exemplaires, nous semble le plus frappant est, sans conteste, la longueur excessive des filaments génitaux. Dans son texte, Root indique : « Genital filaments long (2,7 times as long as lower clasper) and slender, ending in fine, sharp points». Or si nous mesurons le «lower clasper de la figure 5 de Root, en reportant cette longueur sur l'échelle indiquée, nous trouvons: $275 \mu \mathrm{m} \times 2,7$ $=742 \mu \mathrm{m}$ pour la longueur totale des filaments génitaux dont une partie seulement figure sur le dessin de Root. Ces chiffres correspondent, à peu de chose près, à ceux donnés par Floch et Abonnenc pour leur Phlebotomus davisi (Floch et Abonnenc, 1952) et par Mangabeira et pour son $P$. rooti. Ils sont légèrement inférieurs à ce que nous avons observé sur nos échantillons en provenance du Brésil (Bélem) et de la Guyane française (Maripasoula).

\section{Matériel examiné}

L'holotype de L. davisi Root, déposé au U.S. National Museum, a été examiné par l'un d'entre nous (Young) qui a constaté l'absence de l'armature génitale.

Deux mâles et deux femelles de l'Etat de Para (Bélem: Apeg forest), un mâle et une femelle de Guyane française (Maripasoula) et un mâle d'Amazonie (Rio Urubu) ont été examinés, mesurés et dessinés. 
Description du mâle :

- Provenance : Apeg forest, Para (Brésil), 06-08-74.

— Taille : $2,14 \mathrm{~mm}$.

-- Tête: Antenne (fig. $1 \mathrm{~b}$ ) : AII $=230 \mu \mathrm{m} ;$ AIv $=84 \mu \mathrm{m}$. Labre-épipharynx $=$ $170 \mu \mathrm{m} ; \mathrm{AIII} / \mathrm{E}=1,3$. Palpe (fig. $1 \mathrm{c}$ ) : longueur totale $=352 \mu \mathrm{m}$; longueur de chaque segment du $1^{\text {er }}$ au $5^{\mathrm{e}}: 30-88-110-54-70 \mu \mathrm{m}$. Long. palpe/AmI $=1,5$. Cibarium (fig. 1 e) : muni de petites dents verticales réparties sans ordre; plage pigmentée petite, arrondie en demi-cercle.

- Thorax: Soies mésanépisternales supérieures: 16-15. Soies mésanépisternales inférieures: 11-12. Sur les autres exemplaines, le nombre de ces soies variait de 15 à 18 pour les supérieures et de 9 à 12 pour les inférieures. Ailes (fig. $l \mathrm{f}$ ) : longueur $=1,6$ $\mathrm{mm}$, largeur max. $=450 \mu \mathrm{m} ; \alpha=400 \mu \mathrm{m}, \beta=190 \mu \mathrm{m}, \delta=90 \mu \mathrm{m}$. Indice alaire $\alpha / \beta$ $=2,2$. Pattes absentes.

- Abdomen: Genitalia (fig. $1 \mathrm{a}-\mathrm{g}$ ) : Coxite $=210 \mu \mathrm{m}$, style $=140 \mu \mathrm{m}$; il porte 5 épines situées au-delà du milieu du segment, dont une terminale et les 4 autres sur le quart apioal à des niveaux différents. Paramène $=140 \mu \mathrm{m}$, portant sur son bord inférieur 21 soies foliacées, régulièrement réparties et diminuant progressivement de longueur de la base vers l'apex et, près de l'extrémité, des soies lisses, plus petites et de tailles inégales. Un bras recourbé en forme de massue et portant quelques soies à son extrémité distale, s'articule à la base du paramère. Lobe latéral $=260 \mu \mathrm{m}$. Valves péniennes, larges à la base, s'amincissant brusquement en un prolongement presque cylindrique. Pompe génitale $=190 \mu \mathrm{m}$. Filaments génitaux $=870 \mu \mathrm{m}$. Rapport FG/ $\mathrm{PG}=4,5$, rapport $\mathrm{FG} /$ lobe latéral $=3,3$. Sur l'exemplaire de Maripasoula $\mathrm{n}^{\circ} 2723$ les mensurations sont peu différentes.

Description de la femelle:

- Provenance : Apeg forest, Para (Brésil), 18-12-72.

- Taille : $2,05 \mathrm{~mm}$.

- Tête : Antenne (fig. $2 \mathrm{~b}$ et e) : AIII $=270 \mu \mathrm{m} ;$ AIV $=110 \mu \mathrm{m}$. Labre-épipharynx $=300 \mu \mathrm{m}$. AmI $/ \mathrm{E}=0,9$. Palpe (fig. $2 \mathrm{c}$ ) : longueur totale $=485 \mu \mathrm{m} ;$ longueur de chaque segment du $1^{\text {er }}$ au $5^{\mathrm{e}}: 45-145-170-45-80 \mu \mathrm{m}$. Long. palpe/AIII $=1,7$. Cibarium (fig. $2 \mathrm{f}$ ) avec 4 dents horizontales aiguës et environ 23 dents verticales dont les 12 médianes sont disposées en deux rangées longitudinales irrégulières. L'arche, bien marquée, traverse la plage pigmentée au niveau de son extrémité antérieure.

- Thorax: Soies mésanépisternales supérieures: 18-18. Soies mésanépisternales inférieures : 9-9. Aile (fig. $2 \mathrm{~d}$ ) : longueur $=1,8 \mathrm{~mm}$, largeur $\max .=600 \mu \mathrm{m} ; \alpha=410$ $\mu \mathrm{m}, \beta=230 \mu \mathrm{m}, \delta=140 \mu \mathrm{m}$. Indice alaire $\alpha / \beta=1,7$. Pattes absentes.

- Abdomen: Spermathèque (fig. 2 a et a') segmentée avec 9-10 anneaux imbriqués : Long. du corps $=50$ à $60 \mu \mathrm{m}$; Conduits individuels $=30$ à $40 \mu \mathrm{m}$; Conduit commun $=220 \mu \mathrm{m}$. 


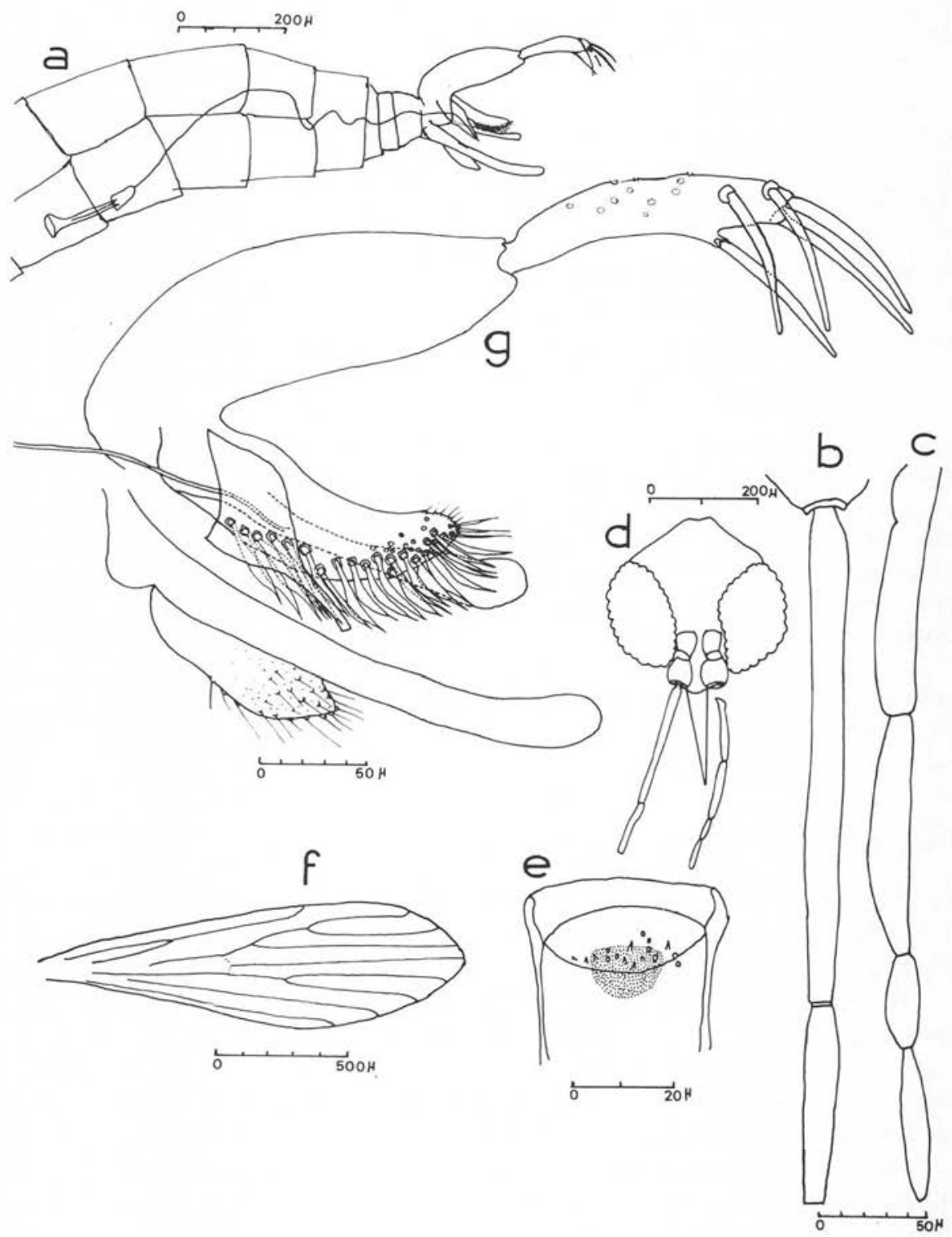

Fig. 1. L. davisi mâle. 


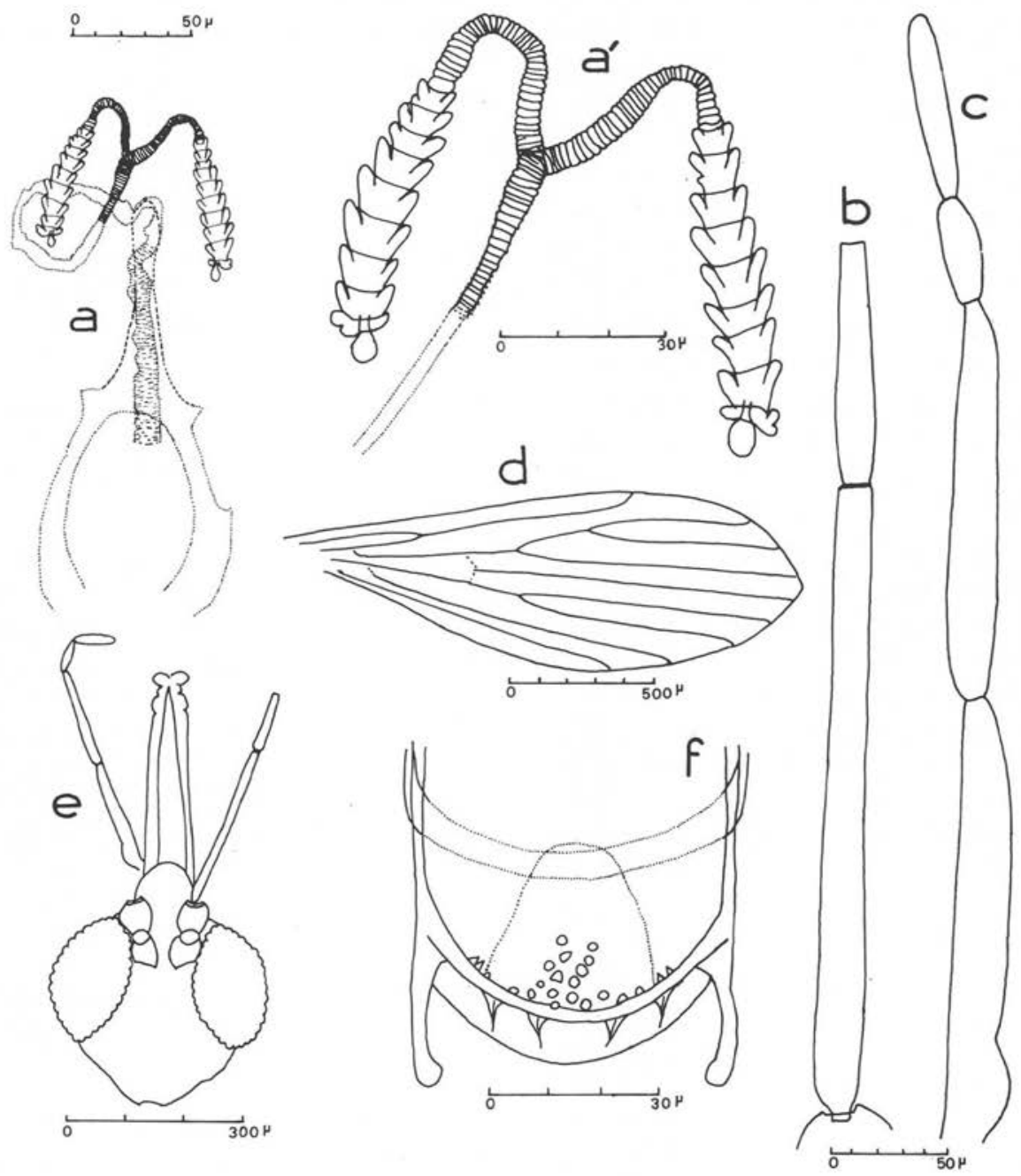

Fig. 2. L. davisi femelle.

\section{Lutzomyia robini n. sp.}

\section{Matériel examiné}

Quatre exemplaires mâles provenant de la même localité : Maripasoula (Guyane française) et capturés au piège lumineux, l'un en mars et les autres en avril. 
Quatre femelles, que nous rapportons à la même espèce, ont été recueillies par le même procédé, 3 dans le même lieu que les mâles et à la même époque, la quatrième sur la rivière Inini en décembre.

Description du mâle :

_ Taille : $2,26 \mathrm{~mm}$

- Tête (fig. $3 \mathrm{e}$ ): Antenne (fig. $3 \mathrm{c}$ ) : AII $=232 \mu \mathrm{m}$, Arv $=100 \mu \mathrm{m}$. Labre-épipharynx $=200 \mu \mathrm{m}$. Am $/ \mathrm{E}=1,16$. Palpe (fig. $3 \mathrm{~d}$ ) : long. totale $=345 \mu \mathrm{m}$; long. de chaque segment du $1^{\text {er }}$ au $5^{\mathrm{e}}: 30-115-50-60 \mu \mathrm{m}$. Long. palpe $/ \mathrm{AmI}=1,4$. Cibarium (fig. 3 b) muni de 16 à 20 petites dents horizontales aiguës, de longueur inégale.

- Thorax: Soies mésanépisternales supérieures: 13-12. Soies mésanépisternales inférieures : 3-3. Sur d'autres exemplaires, le nombre de ces soies variait de 10 à 13 pour les supérieures et de 2 à 4 pour les inférieures. Aile (fig. $3 \mathrm{f}$ ) : long. $=1,75 \mathrm{~mm}$, larg. $\max .=500 \mu \mathrm{m} ; \alpha=400 \mu \mathrm{m}, \beta=200 \mu \mathrm{m}, \delta=50 \mu \mathrm{m}$. Indice alaire $\alpha / \beta=2$. Pattes absentes.

- Abdomen: Genitalia (fig. 3 a et $\mathrm{g}$ ) : Coxite $=200 \mu \mathrm{m}$, style $=145 \mu \mathrm{m}$; il porte 5 épines situées au-delà du milieu du segment: l'une est tarminale, les quatre autres sont insérées sur le tiers distal, à des niveaux différents. Paramère $=130$ à $150 \mu \mathrm{m}$, portant sur son bord postérieur de 20 à 22 soies foliacées, régulièrement réparties et diminuant progressivement de taille de la base vers l'apex. Un bras recourbé, en forme de massue, s'articule à la base du paramère : il porte à son extrémité distale quelques petites soies lisses, plus ou moins longues. Lobe latéral $=280 \mu \mathrm{m}$. Valves péniennes, larges à la base, s'amincissant brusquement vers l'extrémité distale. Pompe génitale $=140 \mu \mathrm{m}$. Filaments génitaux $=440 \mu \mathrm{m}$. Rapport FG/PG $=3,14$, rapport FG/lobe latéral $=1,5$.

Description de la femelle:

- Provenance : Maripasoula (Guyane frảnçaise), capturée en avril, au piège lumineux, $\mathrm{n}^{\circ} 2962$.

— Taille : $2,85 \mathrm{~mm}$.

- Tête: Antenne (fig. $4 \mathrm{c}$ ) : AIII $=245 \mu \mathrm{m}$, Arv $=105 \mu \mathrm{m}$. Labre-épipharynx $=350$ $\mu \mathrm{m}$. AIII/E $=0,67$. Palpe (fig. $4 \mathrm{~b}$ ) : long. totale $=523 \mu \mathrm{m}$; long. de chaque segment du $1^{\text {er }}$ au $5^{\circ}=50-160-188-55-70 \mu \mathrm{m}$. Long. palpe $/ \mathrm{AmI}=2,13$. Cibarium (fig. $4 \mathrm{e}$ ) muni de quatre dents horizontales aiguës, équidistantes, et de 18 petites dents verticales disposées sur deux lignes transverses, plus ou moins régulières. L'arche, bien marquée, traverse le cibarium au niveau du sommet arrondi de la plage pigmentée qui est de forme conique.

- Thorax: Soies mésanépisternales supérieures: 12-12. Soies mésanépisternales inférieures : 4-3. Ailes (fig. $4 \mathrm{f}$ ) : long. $=2,25 \mathrm{~mm}$, larg. $\max .=650 \mu \mathrm{m} ; \alpha=550 \mu \mathrm{m}$, $\beta=250 \mu \mathrm{m}, \delta=120 \mu \mathrm{m}$. Indice alaire $\alpha / \beta=2,2$. Pattes : long. antérieure $=4,03$ $\mathrm{mm}$, médiane incomplète, postérieure $=4,49 \mathrm{~mm}$. 


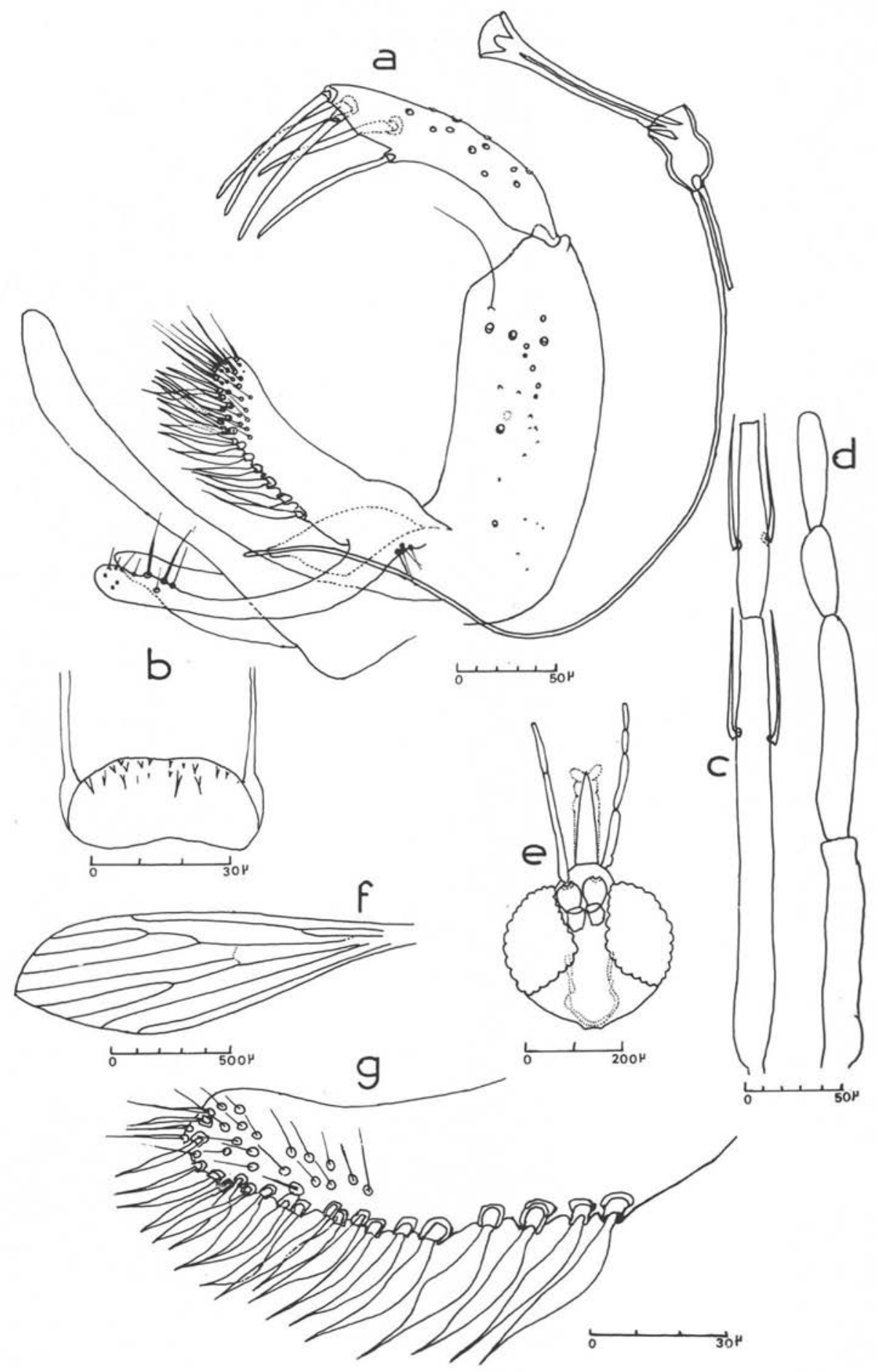

Fig. 3. L. robini mâle. 


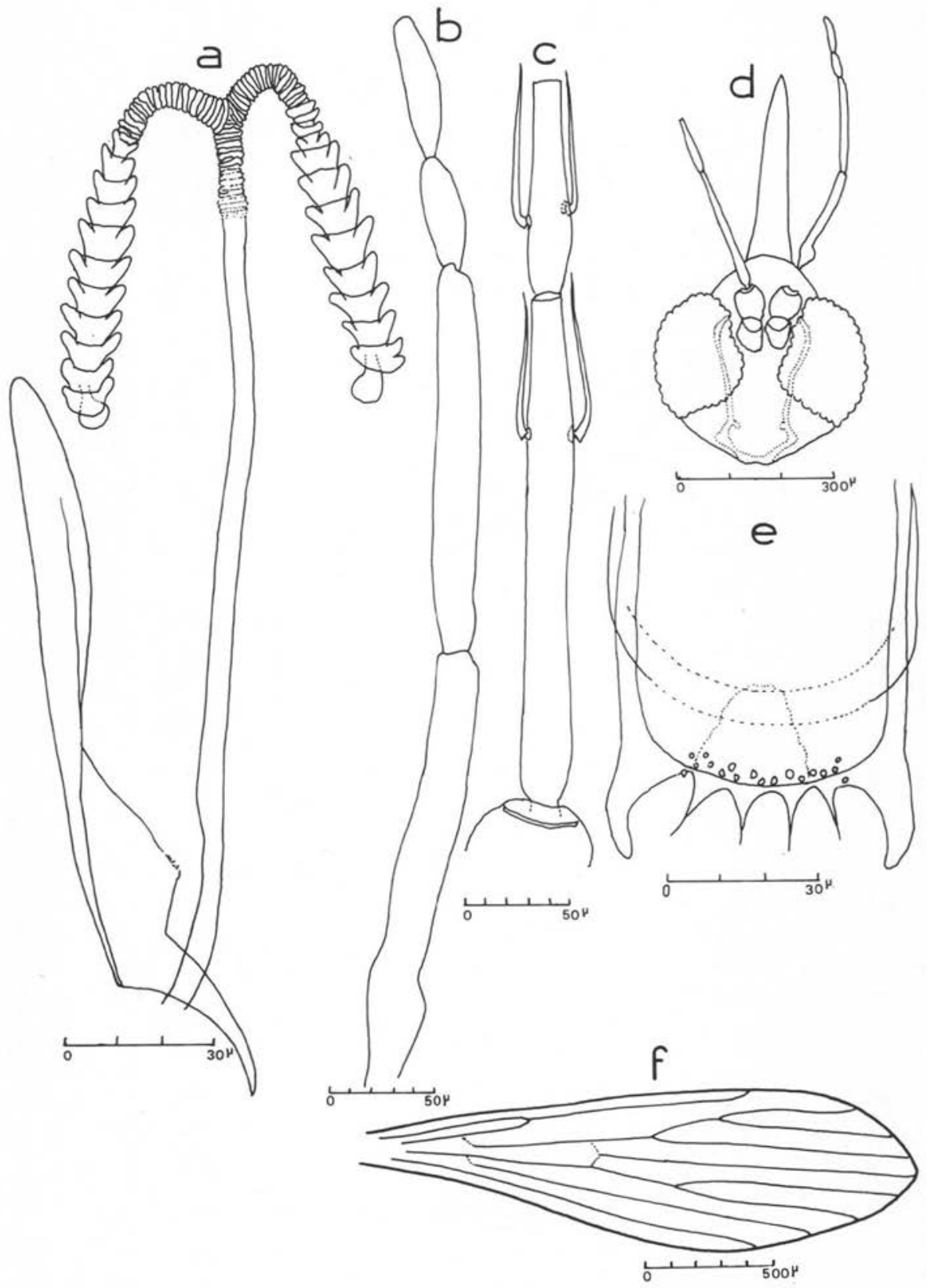

Fig. 4. L. robini femelle. 
- Abdomen : Spermathèque (fig. 4 a) segmentée, avec 10 anneaux imbriqués. Long. $\mathrm{du}$ corps $=55$ à $60 \mu \mathrm{m}$; Conduits individuels $=25$ à $26 \mu \mathrm{m}$; Conduit commun $=$ $185 \mu \mathrm{m}$.

\section{Lutzomyia claustrei Abonnenc, Léger et Fauran, 1979}

Lors de la description récente de cette espèce, nous comparions le mâle à un autre mâle đu même groupe que nous appelions Lutzomyia rooti (Mangabeira, 1942) $[=$ L. davisi (Root, 1934) (Young, 1949) ]. Or un examen approfondi de ce dernier exemplaire nous a permis de rectifier notre diagnose : il s'agissait en fait de $L$. robini que nous venons de décrire.

Nous rappelons ici les principaux caractères de $L$. claustrei mâle.

- Taille: 1,5 à $2 \mathrm{~mm}$.

- Tête : Antenne : $\mathrm{AmI}=270$ à $290 \mu \mathrm{m}, \mathrm{Arv}=96$ à $100 \mu \mathrm{m}$. Labre-épipharynx $=$ 170 à $190 \mu \mathrm{m}$. AIII/E $=1,5$. Palpe : long. totale $=300$ à $350 \mu \mathrm{m}$.

- Thorax: Soies mésanépisternales comptées sur 28 mâles:

\begin{tabular}{|c|c|c|c|c|}
\hline & & Minimum & Maximum & Moyenne \\
\hline supérieures & $\ldots \ldots \ldots \ldots \ldots \ldots$ & 9 & 18 & 12,5 \\
\hline nférieu & $\ldots \ldots \ldots \ldots \ldots \ldots$ & 4 & 9 & 6,6 \\
\hline
\end{tabular}

- Abdomen : Genitalia : Coxite $=190 \mu \mathrm{m}$, style $=138-140 \mu \mathrm{m}$. Paramère $=120$ à $155 \mu \mathrm{m}$, portant sur son bord inférieur une rangée de 14 soies carénées et légèrement foliacées dont les deux médianes sont écartées l'une de l'autre et nettement isolées des autres. Fourreau pénien largement conique, à extrémité mousse, brusquement rétrécie. Lobe latéral $=240$ à $290 \mu \mathrm{m}$. Filaments génitaux $=440$ à $470 \mu \mathrm{m}$. Pompe génitale $=125$ à $140 \mu \mathrm{m}$. Rapport $\mathrm{FG} / \mathrm{PG}=3,3$ à $3,5$.

Description de la femelle présumée de $\mathrm{L}$. claustrei :

- Provenance : Maripasoula (Guyane française), n 2939 capturée le 20-04-72.

- Taille: $2,87 \mathrm{~mm}$.

- Tête (fig. $6 \mathrm{e}$ ) : Long. (y compris le clypeus) $=420 \mu \mathrm{m}$. AmI $=250 \mu \mathrm{m}$, AIv $=$ $95 \mu \mathrm{m}$. Labre-épipharynx $=320 \mu \mathrm{m}$. AIII/ $\mathrm{E}=0,78$. Palpe (fig. $6 \mathrm{c}$ ) : long. totale $=$ $470 \mu \mathrm{m}$, long. de chaque segment du $1^{\text {er }}$ au $5^{\circ}: 50-140-170-40-70 \mu \mathrm{m}$. Long. palpe/ AIII $=1,86$. Cibarium (fig. $6 \mathrm{f}$ ), avec 4 dents horizontales aiguës et 17 dents disposées en une rangée semi-circulaire, sur le bord antérieur de la cavité buccale, les deux dents paramédianes plus grosses que les autres. Arche bien marquée traversant la plage pigmentée au niveau de son sommet.

- Thorax: Soies mésanépisternales comptées sur 18 femelles :

$\begin{array}{lcccc} & & \text { Minimum } & \text { Maximum } & \text { Moyenne } \\ \text { supérieures } & \ldots \ldots \ldots \ldots \ldots \ldots & 10 & 18 & 13,8 \\ \text { inférieures } & \ldots \ldots \ldots \ldots \ldots \ldots & 3 & 9 & 6,8\end{array}$

- Abdomen: Spermathèque (fig. 6 a et a') segmentée, avec 9 anneaux imbriqués : Long. du corps $=48$ à $55 \mu \mathrm{m}$; Conduits individuels $=25$ à $30 \mu \mathrm{m}$; Conduit commun $=130 \mu \mathrm{m}$. 


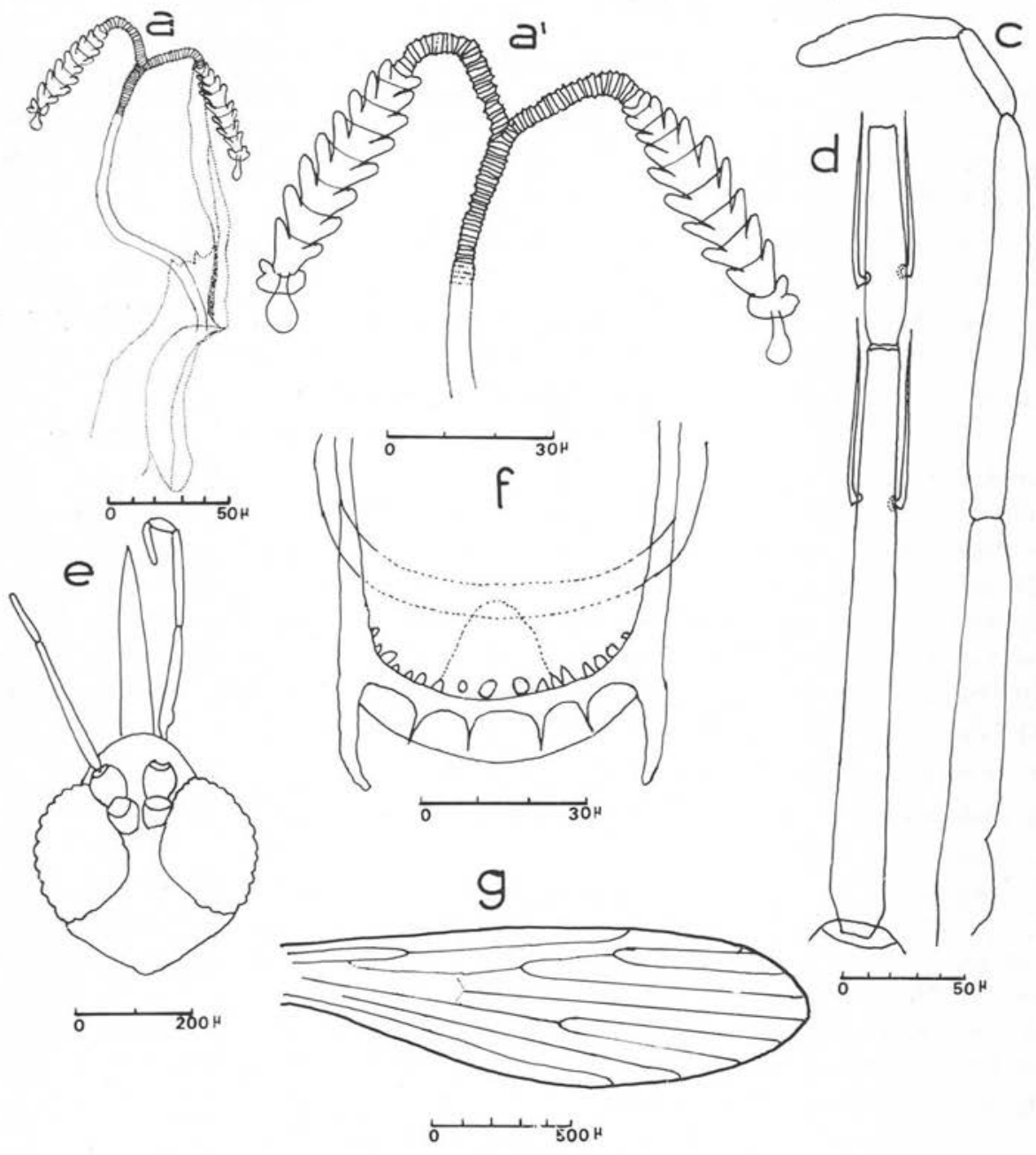

Fig. 5. L. claustrei femelle. 


\section{Dépôt des types.}

La collection type de $L$. claustrei et de L. robini provient de Maripasoula (Guyane française). Elle comprend 4 préparations qui ont été déposées dans les collections du Muséum National d'Histoire Naturelle, section Entomologie générale appliquée, 45, rue de Buffon, 75005 Paris, sous les numéros suivants :

L. claustrei $\mathrm{n}^{\circ} 3428$, holotype mâle, prépar. $33 \mathrm{C}$ du 02-01-73 ;

» $\mathrm{n}^{\circ}$ 3429, allotype femelle, prépar. 2939 du 20-04-72.

L. robini $\mathrm{n}^{\circ}$ 3430, holotype mâle, prépar. 2892 du 20-04-72;

» $\mathrm{n}^{\circ} 3431$, allotype femelle, prépar. 2962 du 20-04-72.

\section{Répartition des 3 espèces}

En Guyane française, ces espèces ont été capturées : dans l'Ile de Cayenne (Cabassou, Paramana, Matoury, Crique Anguille), sur la Comté (Cacao), sur le Maroni (Maripasoula), sur l'Oyapock et sur la rivière Inini.

Au Brésil, le détail des captures s'établit comme suit :

\section{- L. robini :}

A - 4 mâles et 12 femelles, Rio Urubu, Highway AM 264 km (Amazonas), 13-14 mars 1979, piège lumineux, J. Arias et al.

B - 1 mâle et 1 femelle, Ducke Reserve à $27 \mathrm{~km}$ à l'est de Manaus (Amazonas), piège lumineux, D.G. Young.

\section{- L. claustrei :}

Matériel recueilli et identifié par D.G. Young, J. Arias, H. Fraiha, R. Freitas, J. Vidal et al.

A - Belem, Maraba, Altamira (Para).

B - Rio Urubu, Manaus, Ducke Reserve, Highway BR 319 (Amazonas).

- L. davisi :

A - Belem, Apeg forest (Para).

B - Rio Urubu (Amazonas).

Les exemplaires brésiliens de ces trois espèces sont conservés à Gainesville et à Manaus.

En résumé :

Nous avons individualisé dans les captures faites d'une part en Guyane française, d'autre part au Brésil, trois espèces de Psychodopygus étroitement apparentées et qui semblent avoir été souvent confondues dans la littérature : Lutzomyia (Psychodopygus) davisi (Root, 1934), L. (P.) claustrei Abonnenc, Léger et Fauran, 1979 et L. (P.) robini n. sp. 
Les critères différentiels sont les suivants :

\section{1 - pour les mâles:}

$a$ - le nombre et l'aspect des soies sur le paramère :

- soies foliacées (19 à 22) en une seule série longitudinale régulière : $L$ davisi et L. robini,

- soies carénées (13 à 15) dont les deux médianes se détachent nettement des autres : L. claustrei ;

$b-$ la longueur des filaments génitaux : FG/lobe latéral :

$=1,5$ à $2,1: L$. robini et $L$. claustrei,

$=2,7$ à $3,3:$ L. davisi;

2 - pour les femelles:

$a$ - la longueur du conduit commun des spermathèques : conduit commun/corps de la spermathèque :

$=4:$ L. davisi,

$=3,2:$ L. robini,

$=2,6:$ L. claustrei

$b$ - la disposition et le nombre des dents verticales de l'armature cibariale : of. figures ;

3 - pour les deux sexes: le nombre moyen des soies mésanépisternales supérieures et inférieures :

\section{Supérieures}

11

13

L. claustrei ................

L. davisi

\section{Inférieures}

10

La concordance des chiffres observés dans les deux sexes pour chacune des trois espèces, confirme l'intérêt de l'utilisation de ce caractère, non seulement au niveau générique (Abonnenc et Léger, 1976), mais aussi pour la différenciation de centaines espèces.

\section{Bibliographic}

Abonnenc E., Léger N.: Sur une classification rationnelle des Diptères Phlebotomidae. Cah. ORS . TOM, sér. Ent. méd. et Parasitol., 1976, 14, 69-78.

Abonnenc E., Léger N., Fauran P. : Sur deux nouveaux Phlébotomes de la Guyane française : Lutzomyia yuilli pajoti n. s. sp. et Lutzomyia claustrei n. sp. Bull. Soc. Path. Exot., 1979, 72, 75-86. 
Floch H., Abonnenc E. : Phlébotomes de la Guyane et des Antilles françaises. Faune de l'Union française XIV. Larose, édit., Paris, 1952, 205 p.

Mangabeira O.: 7a contribuçao ao estudo dos Flebotomus. Descriçao dos machos de 24 novos especies. Mem. Inst. Oswaldo Cruz, 1942, 37, 111-218.

Root M.: Some american species of Phlebotomus with short terminal segments. Ann. Hyg., 1934, 20, 233-246.

Young D.G.: A review of the bloodsucking Psychodid flies of Colombia (Diptera, Phlebotomina and Sycoracinae). Inst. Food Agric. Sc., Bull. 806, 1979 (technical), Gainesville. 